\title{
Influence of dual-task on postexercise facilitation: a transcranial magnetic stimulation study
}

\author{
Carmen Concerto ${ }^{1, \dagger}$, Bahaa Amer ${ }^{2, \dagger}$, Anaida Abagyann ${ }^{2,}$, Yisheng $\mathrm{CaO}^{2}$, Carmenrita Infortuna ${ }^{2}$, Eileen Chusid ${ }^{2}$, Diego Coira ${ }^{3}$, \\ Fortunato Battaglia ${ }^{1,3, *}$ \\ 1'Department of Interprofessional Health Sciences \& Health Administration, School of Health and Medical Sciences, Seton Hall University, South Orange, NJ, USA \\ 2Department of Pre-clinical Sciences, New York College of Podiatric Medicine, New York, NY, USA \\ ${ }^{3}$ Department of Psychiatry and Behavioral Medicine, Hackensack University Medical Center, Hackensack, NJ, USA
}

In this study we investigated the effect of a dual task (DT) comprised of a nonfatiguing leg and foot extension coupled with a calculation task on postexercise facilitation (PEF) of motor evoked potentials (MEPs) tested by using transcranial magnetic stimulation (TMS). Twelve right-handed healthy subjects participated in the study. They were required to perform a motor task, a cognitive task and a DT. The motor task consisted of extending the right leg and foot for $30 \mathrm{sec}$ at $20 \%$ of the maximal voluntary contraction. The cognitive task consisted of a 30-sec backward calculation. In the DT condition, motor and cognitive tasks were performed concurrently. Resting motor threshold and 10 MEPs were collected before and immediately after each task. TMS was delivered to the motor hot spot of the right vastus lateralis and tibialis anterior (TA) muscles. Results showed that exercise induced a significant PEF in both VL and TA muscles while calculation was not associated with significant PEF. Furthermore, DT was associated with lack of significant PEF in both muscles (VL, $116.1 \% \pm 9.6 \%$; TA, $115.7 \% \pm 9 \%$ ). Our data indicates DT interference on corticospinal excitability after a nonfatiguing exercise. Our experimental paradigm may be used to address postexercise motor cortex plastic adaptations induced by motor and cognitive tasks of different complexity in sport, aging and neuropsychiatric diseases.

Keywords: Dual task, Transcranial magnetic stimulation, Postexercise facilitation, Exercise

\section{INTRODUCTION}

A brief, nonfatiguing exercise has transient and profound effects on cortical function (Lambourne et al., 2010) behavioral and mental performances (Lambourne and Tomporowski, 2010). Postexercise cortical activity has been previously investigated by using different techniques. Indeed, electroencephalographic findings in healthy adults have shown postexercise changes in brain oscillatory activity that correlate with the motor performance (Tsai et al., 2014; Wen et al., 2014). Furthermore previous transcranial magnetic stimulation (TMS) studies provided significant information. TMS is a noninvasive neurophysiological technique that allows us to study cortical excitability and plasticity. A single TMS pulse applied over the primary motor cortex (M1) through the scalp elicits a motor evoked potential (MEP) in the contralateral target muscles. MEP amplitude has been tested as a useful index to investigate corticospinal changes during execution and control of movements (Hallett, 2000, 2007). Notably, TMS studies have shown an increase in amplitudes of MEPs after nonfatiguing exercise, a phenomenon called postexercise facilitation (PEF) (Balbi et al., 2002; Chye et al., 2010; Sacco et al., 1997; Samii et al., 1996). This MEP facilitation is a durable individual characteristic of normal subjects which is not influenced by age or sex and decays to baseline over 2 to $4 \mathrm{~min}$ (Pridmore et al., 2001). If the exercise is repeated until fatigue, MEP amplitude decreases (postexercise depression) (Brasil-Neto et al., 1993; Brasil-Neto et al., 1994). It has been suggested that the postexercise adaptations in MEP size are linked to cortical long-term potentiation/depression, Longterm potentiation/long-term depression-like phenomena within motor cortex and are considered a marker of exercise-induced pri-

This is an Open Access article distributed under the terms of the Creative Commons Attribution Non-Commercial License (http://creativecommons.org/licenses/by-nc/4.0/) which permits unrestricted non-commercial use, distribution, and reproduction in any medium, provided the original work is properly cited.
*Corresponding author: Fortunato Battaglia (D) http://orcid.org/0000-0001-5744-0953 Department of Interprofessional Health Sciences \& Health Administration, School of Health and Medical Sciences, Seton Hall University, 400 South Orange Avenue, South Orange, NJ, 07079, USA

Tel: +1-212-410-8072, Fax: +1-212-410-0940, E-mail: fortunato.battaglia@shu.edu ${ }^{\dagger}$ These authors contributed equally to this study and should be considered co-first authors. Received: May 17, 2016 / Accepted: June 3, 2016 http://www.e-jer.org | pISSN 2288-176X eISSN 2288-1778 
mary motor cortex (M1) plasticity (Samii et al., 1996; Zanette al., 1995).

A growing body of evidence suggests that when compared to a single motor task alone, the addition of a cognitive task (dual task, DT) results in increased performance error and prolongation of reaction time (Corp et al., 2014; Pashler, 1994). This DT interference effects have been interpreted in terms of a fixed-capacity resource model of attention: when the resources needed to perform two tasks concurrently exceed the total capacity there is a decrease in motor performance (Hiraga et al., 2009). However, the majority of studies have employed designs tailored to examine the activation of cortical areas during DT and the effect on motor performance. As a result, it remains unclear if the corticospinal adaptations following a nonfatiguing muscle contraction (PEF) might be modulated by a concurrent cognitive task. Determining whether DT might interfere with the development of PEF will provide an additional perspective about the interplay between exercise and cognition in M1 immediately after an acute exercise.

The objective of this study was to employ TMS and a well-established paradigm to investigate the effect of a DT comprised of a nonfatiguing leg or foot extension coupled with a calculation task on corticospinal excitability tested immediately after the exercise (PEF) in healthy subjects. We hypothesized that DT interference might lead to abnormal postexercised-induced cortical plasticity.

\section{MATERIALS AND METHODS}

\section{Subjects}

Twelve right-handed healthy subjects ( 7 men, 5 women; age range, $22-45 \mathrm{yr}$; mean age, $29.6 \pm 3.7 \mathrm{yr}$ ) participated in the study. The exclusion criteria were head trauma, history epilepsy, use of psychotropic drug and substance abuse. All volunteers gave written informed consent. The study was conducted in accordance with the Declaration of Helsinki and was approved by the Institutional Review Board of the New York College of Podiatric Medicine (approval number: 09/01).

\section{EMG recording}

Surface electromyography (EMG) was recorded with disposable adhesive disk electrodes placed in a tendon-belly arrangement over the right vastus lateralis (VL) and the right tibialis anterior (TA) muscles. The signal was amplified, filtered (bandpass 2-5 $\mathrm{kHz}$ ), digitized (Micro 1401, Cambridge Electronics Design, Cambridge, UK), and stored in a laboratory computer for off-line analysis. During the experiments EMG activity was continuously monitored by visual (oscilloscope) and auditory (speakers) feedback to ensure complete relaxation after the tasks. However, trials where background EMG activity exceeded $0.1 \mathrm{mV}$ were excluded.

\section{Transcranial magnetic stimulation}

Transcranial magnetic stimulation (TMS) was performed with a 9-cm figure-of-eight coil and a Magstim 200 stimulator (The Magstim Company, Dyfed, UK). The coil was placed at the optimal position for eliciting MEPs from the right VL and TA muscles. To produce a predominantly transsynaptic activation of the cortico-spinal neurons the coil was held tangentially to the skull with the handle pointing backward and laterally at an angle of $45^{\circ}$ to the sagittal plane (Rothwell, 1999). We first determine resting motor threshold (RMT), defined as the minimum stimulator intensity (to the nearest $1 \%$ ) to produce an MEP of $50 \mu \mathrm{V}$ in five of 10 trials. We then recorded MEPs obtained using a stimulus intensity of $120 \%$ of the RMT. Mean peak-to-peak MEP amplitudes were determined by averaging 10 monophasic magnetic stimuli delivered to the motor hot spot of the VL and TA muscles.

\section{Experimental procedure}

Subjects were comfortably seated on a chair. Using Velcro stripes, the right leg was secured to the chair to limit unwanted movement during leg and foot extension. PEF after nonfatiguing $\mathrm{VL}$ and TA exercise was investigated on two different days (in a randomized order). Briefly, after a "Go" signal the volunteers were asked to perform a right leg or foot extension as hard as possible to determine the maximal voluntary contraction (MVC). Force

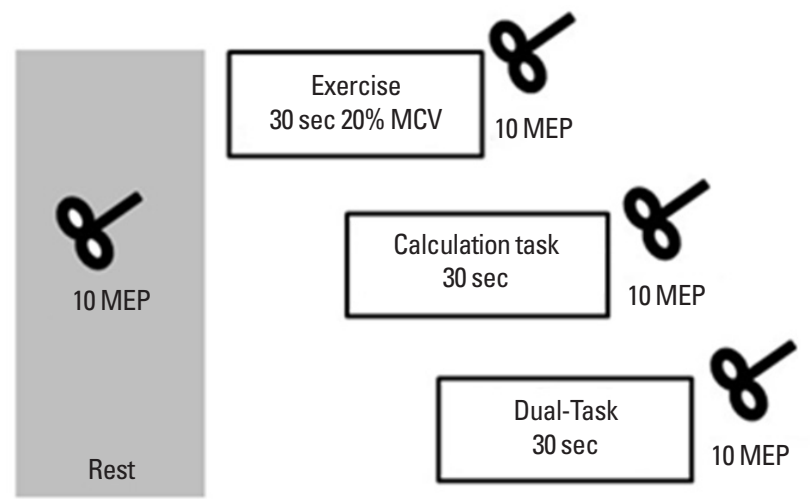

Fig. 1. Experimental design. Ten motor evoked potentials (MEPs) were collected before and immediately after a motor task (MT), a cognitive task (CT) and a dual task (DT). During the MT task subjects performed a leg or foot extensions for $30 \mathrm{sec}$ at $20 \%$ of the maximal voluntary contraction (MCV). The CT consisted of a 30-sec backward calculation. In the DT condition, motor and cognitive tasks were performed concurrently for $30 \mathrm{sec}$. 
output was monitored with a dynamometer attached to a wooden frame (Hoggan Health Industries, West Jordan, UT, USA). Subjects were then required to perform a motor task, a cognitive task and a DT. The motor task consisted of extending the leg or foot for 30 sec at 20\% of their MVC. This motor task has been previously showed to enhance MEPs without significant fatigue (10). The cognitive task consisted of a 30-sec backward calculation (100-3-3.....). In the DT condition motor and cognitive tasks were performed concurrently. Ten MEPs were collected before and immediately after each task (Fig. 1). The three different tasks were performed in the same day in a randomized order with an interval of at least 1 hr between tasks.

\section{Statistical analysis}

Data were analyzed using a mixed model repeated-measures analysis of variance (ANOVA); factors Time (MEP pre, MEP post) Muscles (VL, TA) and tasks (motor, cognitive, DT). The Mauchly test was used to evaluate the assumption of sphericity, and in the case of significant violations, we applied the Greenhouse-Geisser correction. Data analysis was performed with IBM SPSS Statistics ver. 20.0 (IBM Co., Armonk, NY, USA). Statistical significance was set at 0.05 . Data are presented as mean \pm standard error.

\section{RESULTS}

All patients completed the study without reporting any significant side effects. RMT recorded in the right VL muscle and in the right TA muscle did not change after tasks: Time $(F[1,66]=0.40$,

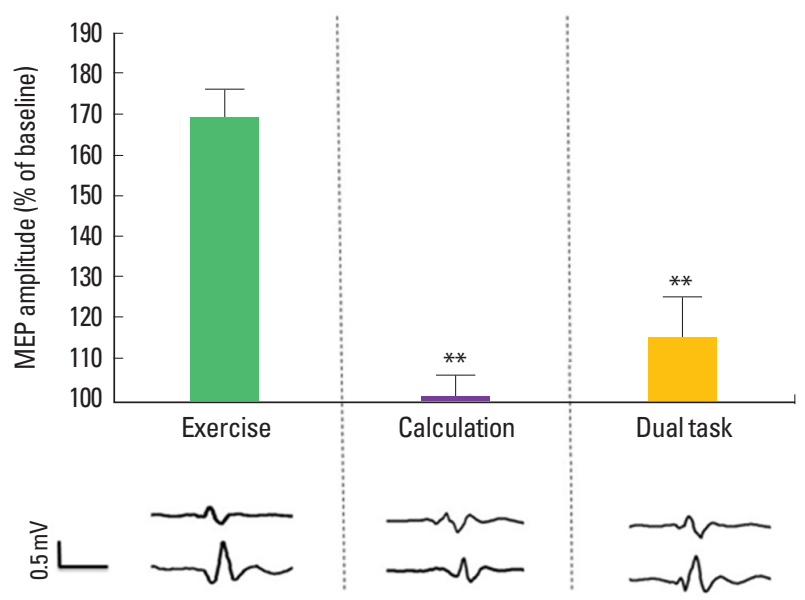

Fig. 2. Pooled percentages of the mean pre-exercise motor evoked potential (MEP) value (post task facilitation) recorded from the vastus lateralis muscle before and after each task. Data are presented as mean \pm standard error. ${ }^{* *} P<0.01$
$P=0.5)$, Muscles $(F[1,66]=1.1, P=0.2)$, Tasks $(F[2,66]=0.06$, $P=0.9$ ) (RMT VL: exercise pre $83.7 \% \pm 2.2 \%$, post $83.6 \% \pm 2.1 \%$; calculation pre $83 \% \pm 2.9 \%$, post $82.8 \% \pm 2.8 \%$; DT pre $85 \% \pm$ $2.6 \%$, post $85.1 \% \pm 2.6 \%$. RMT TA: exercise pre $78.6 \% \pm 3.4 \%$, post $78.8 \% \pm 3.1 \%$; calculation pre $77.5 \% \pm 2.9 \%$, post $78 \% \pm$ $2.9 \%$; DT pre $78.1 \% \pm 3.2 \%$, post $78.3 \% \pm 3 \%$ ).

We then tested the effect of different tasks on PEF. Mixed model repeated measure ANOVA showed a significant effect of time ( $F[1$, 66 $]=48.6, P<0.0001)$ and tasks $(F[2,66]=4.09, P<0.02)$ without a significant effect of muscles $(F[1,66]=0.003, P=0.9)$. There was a significant Time $\times$ Tasks interaction $(F[2,66]=21.46, P<0.0001)$ and a nonsignificant Muscles $\times$ Tasks $\times$ Time $(F[2,66]=0.313$, $P=0.7)$ interaction indicating that different tasks induced similar PEF changes in both muscles. Post hoc analyses showed that for VL MEPs motor task induced a significant increment in MEP size (pre, $0.49 \pm 0.05 \mathrm{mV}$; post, $0.83 \pm 0.09 \mathrm{mV} ; P=0.0002$ ), while both cognitive task (pre, $0.46 \pm 0.02 \mathrm{mV}$; post, $0.47 \pm 0.029 \mathrm{mV} ; P=0.8$ ) and DT (pre, $0.49 \pm 0.05 \mathrm{mV}$; post, $0.57 \pm 0.05 \mathrm{mV} ; P=0.2$ ) did not induce increase in MEP size (Fig. 2). MEP recorded from TA muscle showed similar results with significant increase of PEF only after motor task (MEP motor task: pre, $0.48 \pm 0.03 \mathrm{mV}$; post, $0.75 \pm 0.05 \mathrm{mV} ; P=0.0001$; cognitive task: pre, $0.45 \pm 0.03 \mathrm{mV}$; post, $0.47 \pm 0.03 \mathrm{mV}$; $P=0.6$; DT: pre, $0.49 \pm 0.06 \mathrm{mV}$; post, $0.56 \pm 0.06 \mathrm{mV} ; P=0.9$ ) (Fig. 3).

\section{DISCUSSION}

To our knowledge, this study is the first to show that a DT has

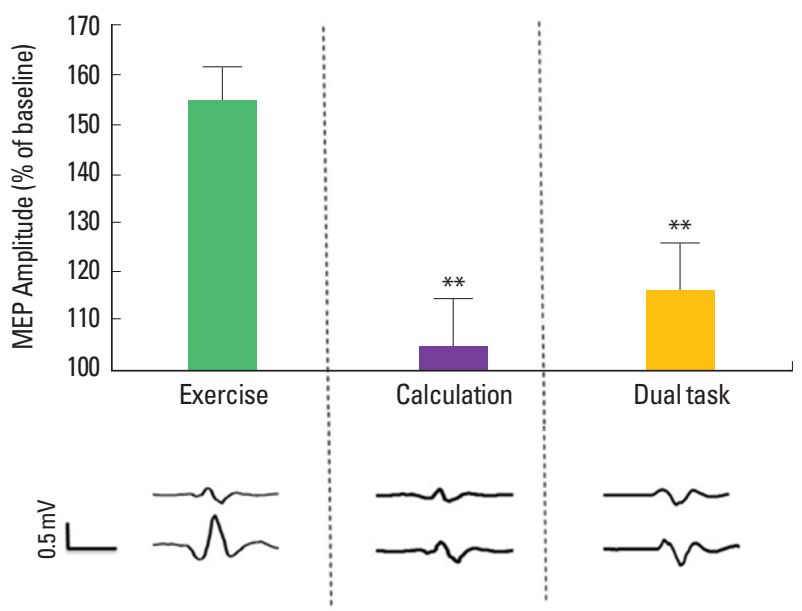

Fig. 3. Pooled percentages of the mean pre-exercise motor evoked potential (MEP) value (post task facilitation) recorded from the tibialis anterior muscle before and after each task. Data are presented as mean \pm standard error. ${ }^{*} P<0.01$. 
a detrimental effect on motor cortex plastic adaptations induced by a brief nonfatiguing exercise.

Previous DT studies focused mostly on complex motor tasks (Strayer and Johnston, 2001) and gait (see a systematic review and meta-analysis) (Al-Yahya et al., 2011). Our results indicate that a concurrent cognitive task interferes with both motor execution and with postexercise corticospinal adaptations. The neural mechanisms underlying the lack of postexercise cortical changes after a DT are still to be investigated. Previous work has shown that PEF cannot be induced with transcranial electrical stimulation (Brasil-Neto, et al., 1994), a technique that stimulates pyramidal neuron at subcortical level. Thus, it is likely that facilitation of MEPs after non-fatiguing exercise reflect plastic intracortical phenomena. (Brasil-Neto, et al., 1993; Samii et al., 1998). Thus, our results might indicate that the increased cognitive load during a DT reduced frontal lobe resource and, consequently prevented motor cortical increases in cortical excitability. This indicates that postexercise corticospinal plastic adaptations engage cognitive networks that are affected by task-irrelevant environmental distracters. Multitasking is an important factor of the executive functions networks (D'Esposito et al.,1995). A number of functional magnetic resonance imaging studies, demonstrated that during $\mathrm{D}$ an increased activity of these networks (particularly in dorsolateral prefrontal cortex) is associated with decreased activation in brain areas involved in motor performance (Erickson et al., 2007). This pattern of activation is consistent with our findings.

The functional relevance of PEF is still unclear. It is likely that PEF might be the result of greater processing demands during exercise and the tendency of the sensorimotor system to maintain the current network set for ongoing, non-fatiguing, actions (Engel and Fries, 2010). Another possible hypothesis indicates that PEF of the corticospinal system might be linked either to the higher level of arousal and changes in neurotransmitters induced by the exercise (Tomporowski, 2003) or to feedback and feed-forward activation of sensory afferents and spinal neurons (Jain et al., 2013; Ludyga et al., 2016). Therefore, it is likely that PEF of MEPs, postexercise cortical changes in $\beta$-band oscillations (Witham and Baker, 2007) and corticomuscular coherence (Larsen et al., 2016) represent temporary corticospinal plastic changes that have positive effects on subsequent task performance. Based on previous work it is conceivable that the higher attentional demands of a DT would have negative effect of exercise-induced sensorymotor plasticity (Rosenkranz and Rothwell, 2006). Therefore, the lack of PEF after DT might represent a new parameter to investigate the complex interaction between exercise, attention and brain plastici- ty. As plastic adaptations to exercise are thought to play a pivotal role in rehabilitation, the DT-related changes in PEF might be relevant in designing effective protocols for patients with gait abnormalities at risk of falling, executive functions deficit and dementia.

\section{CONFLICT OF INTEREST}

No potential conflict of interest relevant to this article was reported.

\section{ACKNOWLEDGMENTS}

The authors thank the volunteers for their contribution to this study.

\section{REFERENCES}

Al-Yahya E, Dawes H, Smith L, Dennis A, Howells K, Cockburn J. Cognitive motor interference while walking: a systematic review and meta-analysis. Neurosci Biobehav Rev 2011;35:715-728.

Balbi P, Perretti A, Sannino M, Marcantonio L, Santoro L. Postexercise facilitation of motor evoked potentials following transcranial magnetic stimulation: a study in normal subjects. Muscle Nerve 2002;25:448452.

Brasil-Neto JP, Cohen LG, Hallett M. Central fatigue as revealed by postexercise decrement of motor evoked potentials. Muscle Nerve 1994;17:713-719.

Brasil-Neto JP, Pascual-Leone A, Valls-Solé J, Cammarota A, Cohen LG, Hallett M. Postexercise depression of motor evoked potentials: a measure of central nervous system fatigue. Exp Brain Res 1993;93:181-184.

Chye L, Nosaka K, Murray L, Edwards D, Thickbroom G. Corticomotor excitability of wrist flexor and extensor muscles during active and passive movement. Hum Mov Sci 2010;29:494-501.

Corp DT, Lum JA, Tooley GA, Pearce AJ. Corticospinal activity during dual tasking: a systematic review and meta-analysis of TMS literature from 1995 to 2013. Neurosci Biobehav Rev 2014;43:74-87.

D'Esposito M, Detre JA, Alsop DC, Shin RK, Atlas S, Grossman M. The neural basis of the central executive system of working memory. Nature 1995;378:279-281.

Engel AK, Fries P. Beta-band oscillations--signalling the status quo? Curr Opin Neurobiol 2010;20:156-165.

Erickson KI, Colcombe SJ, Wadhwa R, Bherer L, Peterson MS, Scalf PE, Kim JS, Alvarado M, Kramer AF. Training-induced functional activation changes in dual-task processing: an FMRI study. Cereb Cortex 2007;17:192-204 
Hallett M. Transcranial magnetic stimulation and the human brain. Nature 2000;406:147-150.

Hallett M. Transcranial magnetic stimulation: a primer. Neuron 2007; 55:187-199.

Hiraga CY, Garry MI, Carson RG, Summers JJ. Dual-task interference: attentional and neurophysiological influences. Behav Brain Res 2009;205:10-18.

Jain S, Gourab K, Schindler-Ivens S, Schmit BD. EEG during pedaling: evidence for cortical control of locomotor tasks. Clin Neurophysiol 2013; 124:379-390.

Lambourne K, Audiffren M, Tomporowski PD. Effects of acute exercise on sensory and executive processing tasks. Med Sci Sports Exerc 2010; 42:1396-1402.

Lambourne K, Tomporowski P. The effect of exercise-induced arousal on cognitive task performance: a meta-regression analysis. Brain Res 2010;1341:12-24.

Larsen LH, Jensen T, Christensen MS, Lundbye-Jensen J, Langberg H, Nielsen JB. Changes in corticospinal drive to spinal motoneurones following tablet-based practice of manual dexterity. Physiol Rep 2016 Feb;4(2). pii: e12684

Ludyga S, Gronwald T, Hottenrott K. The athlete's brain: cross-sectional evidence for neural efficiency during cycling exercise. Neural Plast 2016;2016:4583674.

Pashler H. Dual-task interference in simple tasks: data and theory. Psychol Bull 1994;116:220-244.

Pridmore S, Bruno R, Turnier-Shea Y, Rybak M, Reid P. Postexercise facilitation appears durable in normal subjects. Psychiatry Clin Neurosci 2001;55:157-159.

Rosenkranz K, Rothwell JC. Spatial attention affects sensorimotor reorganisation in human motor cortex. Exp Brain Res 2006;170:97-108.

Rothwell JC. Paired-pulse investigations of short-latency intracortical fa- cilitation using TMS in humans. Electroencephalogr Clin Neurophysiol Suppl 1999;51:113-119.

Sacco P, Thickbroom GW, Thompson ML, Mastaglia FL. Changes in corticomotor excitation and inhibition during prolonged submaximal muscle contractions. Muscle Nerve 1997;20:1158-1166.

Samii A, Chen R, Wassermann EM, Hallett M. Phenytoin does not influence postexercise facilitation of motor evoked potentials. Neurology 1998:50:291-293

Samii A, Wassermann EM, Ikoma K, Mercuri B, Hallett M. Characterization of postexercise facilitation and depression of motor evoked potentials to transcranial magnetic stimulation. Neurology 1996;46:13761382.

Strayer DL, Johnston WA. Driven to distraction: dual-Task studies of simulated driving and conversing on a cellular telephone. Psychol Sci 2001;12:462-466.

Tomporowski PD. Effects of acute bouts of exercise on cognition. Acta Psychol (Amst) 2003;112:297-324.

Tsai CL, Chen FC, Pan CY, Wang CH, Huang TH, Chen TC. Impact of acute aerobic exercise and cardiorespiratory fitness on visuospatial attention performance and serum BDNF levels. Psychoneuroendocrinology 2014;41:121-131.

Wen CP, Wai JP, Tsai MK, Chen CH. Minimal amount of exercise to prolong life: to walk, to run, or just mix it up? J Am Coll Cardiol 2014;64: $482-484$.

Witham CL, Baker SN. Network oscillations and intrinsic spiking rhythmicity do not covary in monkey sensorimotor areas. J Physiol 2007; 580(Pt 3):801-814.

Zanette G, Bonato C, Polo A, Tinazzi M, Manganotti P, Fiaschi A. Long-lasting depression of motor-evoked potentials to transcranial magnetic stimulation following exercise. Exp Brain Res 1995;107:8086. 